\title{
Combined Correction of Experimental Critical Ischemia of the Lower Extremities in a Rat Model
}

\author{
Elvin E. Feyziev ${ }^{1}$, Boris S. Sukovatykh ${ }^{1}$, Alexander S. Belous ${ }^{2}$, Maria A. Zatolokina ${ }^{1}$, \\ Elena V. Trubnikova², Ekaterina S. Mishina ${ }^{1 *}$ \\ ${ }^{1}$ Kursk State Medical University \\ ${ }^{2}$ Kursk State University \\ Kursk, the Russian Federation
}

\begin{abstract}
The purpose of our research was to study the effectiveness of correcting experimental critical ischemia (CI) of the lower extremities with a combination of udenafil, simvastatin, and autologous bone marrow mononuclear cells (ABMMC).

Methods and Results: The experiments were carried out on 24-month-old Wistar rats, weighing 220-250g. The animals were randomized by sex and weight. Groups were formed according to the manipulations carried out during the operations. The animals were divided into 7 groups, each with 20 animals: Group 1 included intact animals; Group 2 - falsely operated animals; Group 3 (control group) - animals with simulated CI without treatment; Group 4- animals with CI and monotherapy with udenafil (daily oral administration of $8.6 \mathrm{mg} / \mathrm{kg}$ for 28 days); Group 5 - animals with CI and simvastatin monotherapy (daily oral administration of $1.71 \mathrm{mg} / \mathrm{kg}$ for 28 days); Group 6 - animals with CI and monotherapy with ABMMC (parenterally, once on Day 7 after modeling CI, $50 \mu \mathrm{l}$ at 4 points and, paravasally, above the inguinal ligament in the area where the lateral artery leaves the artery enveloping the femur from the internal iliac artery; in the area of the superficial artery that bends around the iliac bone under the inguinal ligament; into the area of origin of the muscular branch of the femoral artery r. muscularis, the place of attachment of the comb and long adductor muscles of the thigh; in the upper third of the gastrocnemius muscle]); Group 7 - animals with CI and combination therapy (udenafil and simvastatin drugs were administered intragastrically $0.86 \mathrm{mg} /$ $\mathrm{kg}$, once a day, for 7 days) and one-time parenteral administration of ABMMC, according to the same scheme as in Group 6. On Days 21 and 28 of the experiment, the level of blood microcirculation was determined in the muscles of the rat leg; for this, laser Doppler flowmetry was used. For further morphometric assessment of the leg muscles, they were removed. Preparations for morphometric analysis were prepared according to the standard technique with Van Gieson staining, as well as H\&E. Our study demonstrated the effectiveness of combination therapy with udenafil, simvastatin, and ABMMC to correct critical lower limb ischemia in rats. The severity of morphological changes on the background of this combination was minimal, compared to the findings of other study groups, and the level of blood microcirculation in the ischemic zone on Day 28 was, significantly, 1.9 times higher than in animals of the control group.

Conclusion: The results obtained allow us to recommend the use of the investigated combination (udenafil+simvastatin+ABMMC) for the treatment of patients with critical limb ischemia, both in outpatient and inpatient practice. (International Journal of Biomedicine. 2021;11(3):372-375.)

Key Words: critical ischemia $\bullet$ microcirculation $\bullet$ autologous bone marrow mononuclear cells

For citation: Feyziev EE, Sukovatykh BS, Belous AS, Zatolokina MA, Trubnikova EV, Mishina ES. Combined Correction of Experimental Critical Ischemia of the Lower Extremities in a Rat Model. International Journal of Biomedicine. 2021;11(3):372375. doi:10.21103/Article11(3)_OA16
\end{abstract}

\section{Abbreviations}

ABMMC, autologous bone marrow mononuclear cells; CG, control group; CI, critical ischemia; EG, experimental group; H\&E, hematoxylin and eosin; PU, perfusion units. 


\section{Introduction}

Because critical ischemia (CI) of the lower extremities leads to disability and death not only in the elderly, but also in people of working age, it is one of the serious diseases of the cardiovascular system. CI requires a timely and accurate assessment of the patient's condition, analysis of the dynamics of the development of this pathology, as well as intensive treatment. ${ }^{(1,2)}$ Most often, surgical methods of treatment are used to treat this pathology; however, there are $20 \%-25 \%$ of patients with diffuse vascular lesions and without a distal vascular bed, for whom the use of this method of treatment is limited. ${ }^{(3,4)}$ In the last decade, phosphodiesterase-5 inhibitors, which were originally used as a means of correcting erectile dysfunction, have been actively studied. They are currently being investigated for further use in cardiology, gynecology, gastroenterology and many other related medical specialties. Phosphodiesterase- 5 inhibitors have vasodilating activity and are effective in correcting skeletal muscle ischemia. ${ }^{(5,6)}$ To correct CI of the lower extremities, the use of drugs of this group, in our opinion, is pharmacoeconomically more expedient than drugs of the group of synthetic prostaglandins. Statins are of particular interest for the prevention and treatment of atherosclerosis and coronary artery disease. In addition to their main activities, the drugs of this group have many pleiotropic effects: improved endothelial function; anti-inflammatory, antiplatelet, antioxidant effects; slowing down the proliferation of smooth muscle cells in the vascular wall; and stimulation of fibrinolysis, all of which do not depend on hypocholesterolemic activity in drugs of this group. These drugs also have an inhibitory effect on the synthesis of pro-inflammatory cytokines:(7) interleukins- $(1,6,8)$ and TNF- $\alpha$. In addition, in patients with hypertriglyceridemia, statins normalize the concentration of triglycerides. ${ }^{(8)}$ There are also studies that have demonstrated experimentally the effectiveness of ABMMC for the treatment of critical limb ischemia. This treatment is effective because ABMMC contains stem cells, such as hematopoietic and mesenchymal. These cells contribute to the replacement of tissue defects and neoangiogenesis since they have multipotent properties..$^{(9)}$ Thus, the use of a combination of udenafil, simvastatin and ABMMC in the case of limb ischemia, acting on different links in the pathogenesis of this pathology, may be completely justified.

The purpose of our research was to study the effectiveness of correcting experimental CI of the lower extremities with a combination of udenafil, simvastatin, and ABMMC.

\section{Materials and Methods}

The experiments were carried out on 24-monthold Wistar rats, weighing 220-250g. Before the study, the experimental animals were quarantined for 7 days in the vivarium of the Research Institute of EM at the Kursk State Medical University. The rats were kept in a standard room with 12 hours of automatic lighting and air temperature maintained at $22-24{ }^{\circ} \mathrm{C}$.

The animals were randomized by sex and weight. Groups were formed according to the manipulations carried out during the operations. The animals were divided into
7 groups, each with 20 animals: Group 1 included intact animals; Group 2 - falsely operated animals; Group 3 (CG) animals with simulated CI without treatment; Group 4 (EG1) - animals with CI and monotherapy with udenafil; Group 5 (EG2) - animals with CI and simvastatin monotherapy; Group 6 (EG3) - animals with CI and monotherapy with ABMMC

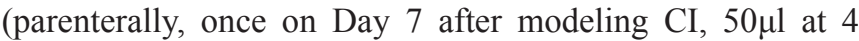
points and, paravasally, above the inguinal ligament in the area where the lateral artery leaves the artery enveloping the femur from the internal iliac artery; in the area of the superficial artery that bends around the iliac bone under the inguinal ligament; into the area of origin of the muscular branch of the femoral artery r. muscularis, the place of attachment of the comb and long adductor muscles of the thigh; in the upper third of the gastrocnemius muscle]); Group 7 (EG4) - animals with $\mathrm{CI}$ and combination therapy (intragastrically udenafil and simvastatin) and one-time parenteral administration of ABMMC, according to the same scheme as in Group 6.

All experimental manipulations were performed in the afternoon. The animals were anesthetized with chloral hydrate, introduced intraperitoneally at a dose of $300 \mathrm{mg} / \mathrm{kg}$ in an aqueous solution that was prepared in advance on the day of the experiment. Before surgery, the rats were fixed on a special table, after which the hair was shaved off on the inner surface of the thigh and lower leg, the incision area was treated with a $70 \%$ aqueous solution of alcohol. The rats were removed from the experiment by means of an overdose of anesthesia. In the group of sham-operated rats, after anesthesia, all experimental animals were operated on through the skin along the surface of the thigh and lower leg from the inside, and the neurovascular bundle was isolated, after which the wound was sutured. The CG was simulated similarly to the sham-operated rats, but with the removal of a portion of the great vessels, such as the femoral, popliteal, anterior, and posterior tibial arteries and veins, as well as the sciatic nerve. ${ }^{(10)}$ In Group 4, the experimental pathology was corrected daily, for 28 days, by oral administration of udenafil $8.6 \mathrm{mg} / \mathrm{kg}$, and in Group 5 - daily, for 28 days, with oral administration of simvastatin $1.71 \mathrm{mg} / \mathrm{kg}$. In Group 6, $\mathrm{CI}$ was corrected by introducing ABMMC (from the tibia by puncture of the bone marrow cavity and cell isolation according to the Boyum method), a single parenteral injection on Day 7 after modeling $\mathrm{CI}, 50 \mu \mathrm{l}$ at 4 points, as described above. In Group 7, CI was corrected with a combination of udenafil and simvastatin (both drugs were administered intragastrically 0.86 $\mathrm{mg} / \mathrm{kg}$, once a day, for 7 days) and a single ABMMC injection, similar to Group 6.

On Days 21 and 28 of the experiment, the level of blood microcirculation was determined in the muscles of the rat leg; for this, laser Doppler flowmetry was used. In our study, we used the TSD144 needle sensor included in the kit for the LDF100C hardware complex. The data were recorded and analyzed using the AcqKnowledge 3.8.1 program included in the BIOPAC Systems. Blood microcirculation indices were measured in perfusion units (PU). After that, for further morphometric assessment of the leg muscles, they were removed. All biomaterial for histology was fixed in $10 \%$ formalin aqueous solution for 7 days. Preparations for morphometric analysis were prepared according to the 
standard technique with Van Gieson staining, as well as H\&E. A Levenhuk 320 microscope (USA) with a Levenhuk C310 digital camera was used for morphometric analysis. The processing was carried out using the ScopeTek ScopePhoto 3.1.268 program. Considering the data of previous studies, the most informative were the results obtained on Day 28. We considered the results that were obtained on Day 21 as additional.

In vivo experiments were carried out in accordance with the legislation of the Russian Federation, in strict compliance with the European Convention for the protection of animals used for experimental and other purposes (Strasbourg, France, 1986), the provisions of Directive 210/63/EU of the European Parliament and the Council of the European Union of 22 September 2010 on the protection of animals used for scientific purposes (Article 27), and approved by the Regional Ethics Committee of Kursk State Medical University (Protocol No. 4 dated June 10, 2019).

Statistical analysis was performed using the STATISTICA software package (v.12.0, Stat-Soft Inc., USA). The mean (M) and standard error of the mean (SEM) were calculated. Student's unpaired and paired t-tests were used to compare two groups for data with normal distribution. Differences of continuous variables departing from the normal distribution, even after transformation, were tested by the Mann-Whitney U-test. The Wilcoxon criterion was used to compare the differences between the paired samples. A value of $P<0.05$ was considered significant.

\section{Results}

In the leg muscles of intact rats on Days 21 and 28 of the experiment, the average microcirculation level was 531.6 \pm 12.1 PU. Microscopic analysis of histological sections of the region of the middle third of the tibia revealed welldefined longitudinally and transversely cut symplasts of skeletal muscle tissue, connective tissue layers forming endo, peri- and epimysium of muscle fibers, and small and large blood vessels. Blood vessels had no pathological changes. In the sarcoplasm of the muscles, transverse striation was clearly visualized, as well as elongated basophilic nuclei of a dark shade (Fig. 1A).

Sham-operated rats showed no significant differences, compared to intact rats, in the structure of muscle tissue and indicators of the level of microcirculation $(523 \pm 13.8 \mathrm{PU}$ on Day $21(P=0.645)$ and $524.1 \pm 15.1 \mathrm{PU}$ on Day $28(P=0.625))$ (Fig.1B). However, the rats of the CG showed significant differences, compared to intact rats $(249.1 \pm 7.3$ PU on Day $21(P<0.05)$ and $302.9 \pm 6.5$ PU on Day $28(P<0.05)$. On Day 21, a high degree of tissue reactivity was observed in the affected muscles. Signs of inflammation were pronounced. In the immediate vicinity of the areas of necrosis, bundles of atrophied muscle fibers were periodically visualized. In the layers between the symplasts of the skeletal muscle, the cellular component prevailed over the fibrous one. Primarily lymphocytes, monocytes, eosinophils, single fibroblasts, and fibrocytes were visualized in the field of view. In the epimysium, tissue surrounding the muscle, there were signs of interstitial edema as well as newly formed blood-filled capillaries with signs of perivascular round-cell infiltration. On Day 28 of the experiment, there was a decrease in reactivity tissues with partial normalization of muscle color; however, they were somewhat hypotrophic. The necrotic areas were somewhat smaller. It should be noted that full-blooded, single capillaries were formed (Fig.1C).
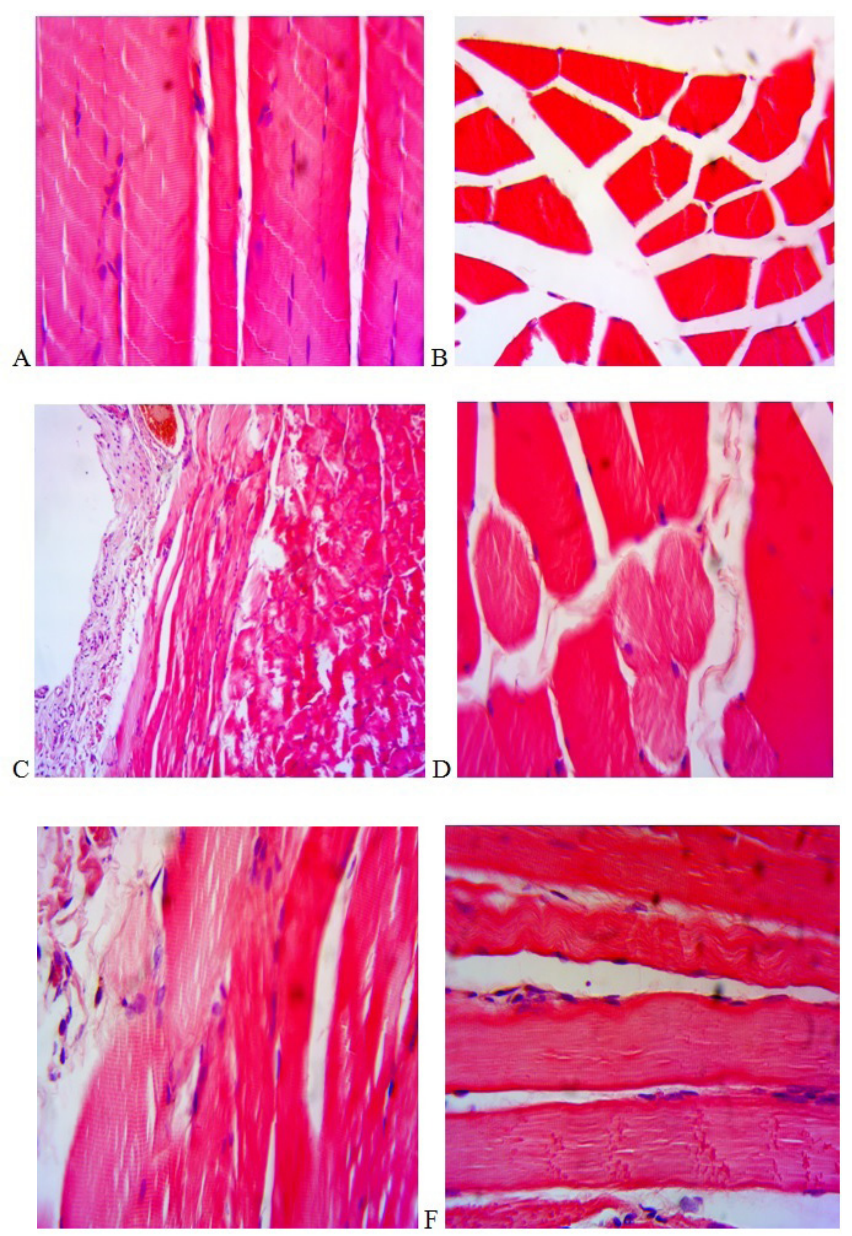

Fig. 1. Micrograph of a section of skeletal muscle tissue in the region of the middle third of the rat leg on Day 28 of the experiment. $H \& E$ staining $(\times 400)$. A-muscles of the leg of the intact rats; $B$ - muscles of the lower leg of the rats of the shamoperated group; $C$-ischemic muscles of the lower leg of the rats of the CG; D - ischemic leg muscles of rats treated with udenafil; $D$ - ischemic leg muscles of rats treated with simvastatin; $E$ - ischemic muscles of the leg of rats after administration of ABMMC; F - ischemic leg muscles of rats treated with udenafil, simvastatin in combination with $A B M M C$.

In Group 4 with udenafil monotherapy, the level of regional blood flow was significantly higher than in the $\mathrm{CG}$ at the corresponding time and was 394.6 \pm 6 PU on Day $21(P<0.05)$ and $486 \pm 7.6 \mathrm{PU}$ on Day $28(P<0.05)$. The level of microcirculation in this group on Days 21 and 28 approached the values of the group of intact animals. Microscopy revealed small foci of atrophying myocytes with cell proliferation, and a decrease in the size and number of necrotic areas due to the formation of young connective tissue. However, there was round-cell infiltration of connective tissue layers between skeletal muscles, small blood vessels (arterioles and venules) were dilated with signs of 
thrombosis, and a new capillary network was formed near the sites of necrosis (Fig.1D). In Group 5, simvastatin monotherapy also had a positive effect on microcirculation indices $(382.6 \pm 4.3$ PU on Day $21(P<0.05)$ and 468.5 \pm 6.8 PU on Day $28(P<0.05)$. The histological picture was similar to the EG1, but the expansion of small blood vessels was less pronounced (Fig.1E). In Group 6 with ABMMC monotherapy, the level of regional blood flow was 401.3 \pm 10.9 PU on Day $21(P<0.05)$ and 504.8 \pm 8.5 PU on Day 28 $(P<0.05)$. As in the EG1 group, microscopy revealed a decrease in the number and size of necrotic areas. Circular cell infiltration of connective tissue layers between skeletal muscles continued, small blood vessels were dilated with signs of thrombosis, and a new capillary network was formed near the sites of necrosis. There were local areas with insufficiently stained sarcoplasm and no transverse striation. The density of cells was high, cells of the fibroblastic series predominated in the field of view, and single lymphocytes were visualized. In the interlayers between the symplasts of hypertrophied mast cells, which were in the stage of secretion accumulation and degranulation (Fig.1E). In the EG4, the level of regional blood flow was $493.4 \pm 17.5 \mathrm{PU}$ on Day 21 $(P<0.05)$ and 574.7 \pm 9.9 PU on Day $28(P<0.05)$. The severity of morphological changes in this group was minimal among all observation groups. Symplasts of muscle fibers have a uniform sarcoplasmic coloration. When imaging at high magnification, both on transverse and longitudinal sections, small elongated nuclei located along the periphery of the muscle fibers were visualized. In the layers of connective tissue, the density of cells was low; in the field of view of the fibroblast prevailed. Mast cells were small, mainly in the stage of secretion accumulation, and lymphocytes were single. Small blood vessels were filled with blood, without signs of structural abnormalities. A large number of newly formed blood vessels with signs of thrombosis were visualized in the wide layers of connective tissue (in the areas between the muscles where large vessels were originally localized). We found large blood vessels without significant structural changes. Endotheliocytes were flat, the cell axis was oriented along the basement membrane, and the muscular membrane was formed by smooth myocytes, without signs of fibrosis (Fig.1F).

In conclusion, our study demonstrated the effectiveness of combination therapy with udenafil, simvastatin, and ABMMC to correct critical lower limb ischemia in rats. The severity of morphological changes on the background of this combination was minimal, compared to the findings of other study groups, and the level of blood microcirculation in the ischemic zone on Day 28 was, significantly, 1.9 times higher than in animals of the CG. The results obtained allow us to recommend the use of the investigated combination (udenafil+simvastatin+ABMMC) for the treatment of patients with critical limb ischemia, both in outpatient and inpatient practice.

*Corresponding author: Ekaterina S. Mishina, PhD. Department of Histology, Embryology, and Cytology. Kursk State Medical University. Kursk, Russia.E-mail: katusha100390@list.ru

\section{Competing Interests} interests.

The authors declare that they have no competing

\section{References}

1. Sumin AN, Medvedeva JuD, Shheglova AV, Barbarash LS. [Predictors of adverse outcomes in patients with atherosclerosis obliterans of the lower limb arteries]. Cardiology and Cardiovascular Surgery. 2020;13(1):41-7. [Article in Russian].

2. Emeljanov SG, Korenevskij NA, Bykov AV. [Predicting the severity of the ischemic process in the heart, brain and lower extremities based on fuzzy models]. Biomedical Radio Eeectronics. 2016;9:4-9. [Article in Russian].

3. Genkel VV, Salashenko AO, Shamaeva TN, Sumerkina VA, Nikushkina KV, Shaposhnik II. [Atherosclerosis of peripheral arteries in patients with coronary artery disease and type 2 diabetes mellitus]. Ter Arkh. 2019 Oct 15;91(10):5462. doi: 10.26442/00403660.2019.10.000106. [Article in Russian].

4. Bogachev VYu, Boldin BV, Rodionov SV, Turkin PYu. Conservative treatment of chronic obliterating diseases of lower limb arteries. Ambulatornaya Khirurgiya $=$ Ambulatory Surgery (Russia). 2018;(1-2):14-19. [Article in Russian].

5. Kondratyeva LV. [Barriers to statin therapy in patients with type 2 diabetes mellitus and ways to overcome them]. Russian Medical Journal. 2017;22:1621-1628. [Article in Russian].

6. Arutyunov GP, Boytsov SA, Voevoda MI, Gurevich VS, Drapkina OM, Kukharchuk VV, et al. [Correction of hypertriglyceridemia in order to reduce the residual risk in diseases caused by atherosclerosis]. Conclusion of the Council of Experts of the Russian Society of Cardiology, the Russian Scientific Medical Society of Physicians, the Eurasian Association of Physicians, the National Society for the Study of Atherosclerosis, the Russian Association of Endocrinologists and the National Research League of Cardiological Genetics. Rational Pharmacotherapy in Cardiology. 2019;15(2):282-8. [Article in Russian].

7. Osipova OS, Saaia SB, Karpenko AA, Zakiian SM. Problemy i perspektivy kletochnor̆ terapii kriticheskor̆ ishemii nizhnikh konechnosteî [Problems and prospects of cell therapy for critical ischaemia of lower limbs]. Angiol Sosud Khir. 2020;26(2):23-33. doi: 10.33529/ANGIO2020220. [Article in Russian].

8. Feyziev EE, Belous AS, Sukovatykh BS, Trubnikova EV. Kursk State University, patent holder. A method for modeling critical ischemia of the lower extremities in experimental rat animals. RF patent, RU 2734158 dated 03/18/2020.

9. Böyum A. Separation of leukocytes from blood and bone marrow. Introduction. Scand J Clin Lab Invest Suppl. 1968;97:7.

10. Kolesnik IM, Lazarenko VA, Pokrovsky MV. [Effect of pharmacological preconditioning with sildenafil and vardenafil on the state of the microvasculature in ischemic skeletal muscle]. Kursk Scientific and Practical Bulletin "Man and His Health.” 2015;(1):83-6. [Article in Russian]. 